\title{
QUEEN'S
UNIVERSITY
BELFAST
}

\section{Experimental tests and signal unfolding of a scintillator calorimeter for laser-plasma characterization}

\author{
Istokskaia, V., Stránský, V., Giuffrida, L., Versaci, R., Grepl, F., Tryus, M., Velyhan, A., Dudžák, R., Krása, J., \\ Krupka, M., Singh, S., Neely, D., Olšovcová, V., \& Margarone, D. (2021). Experimental tests and signal \\ unfolding of a scintillator calorimeter for laser-plasma characterization. Journal of Instrumentation, 16(2), \\ [T02006]. https://doi.org/10.1088/1748-0221/16/02/T02006
}

Published in:

Journal of Instrumentation

Document Version:

Peer reviewed version

Queen's University Belfast - Research Portal:

Link to publication record in Queen's University Belfast Research Portal

Publisher rights

Copyright 2021 IOP Publishing Ltd and Sissa Medialab.

This work is made available online in accordance with the publisher's policies. Please refer to any applicable terms of use of the publisher.

\section{General rights}

Copyright for the publications made accessible via the Queen's University Belfast Research Portal is retained by the author(s) and / or other copyright owners and it is a condition of accessing these publications that users recognise and abide by the legal requirements associated with these rights.

Take down policy

The Research Portal is Queen's institutional repository that provides access to Queen's research output. Every effort has been made to ensure that content in the Research Portal does not infringe any person's rights, or applicable UK laws. If you discover content in the Research Portal that you believe breaches copyright or violates any law, please contact openaccess@qub.ac.uk. 


\title{
Experimental tests and signal unfolding of a scintillator calorimeter for laser-plasma characterization
}

\author{
V. Istokskaia ${ }^{\mathrm{a}, \mathrm{b},{ }^{*}, \text { V. Stránský }}{ }^{\mathrm{a}, \mathrm{b}}$, L. Giuffrida ${ }^{\mathrm{b}}$, R. Versaci $^{\mathrm{b}}$, F. Grepl $^{\mathrm{a}, \mathrm{b}}$, M. Tryus $^{\mathrm{b}}$, \\ A. Velyhan $^{\mathrm{b}}$, R. Dudžák ${ }^{\mathrm{c}}$, J. Krása $^{\mathrm{c}}$, M. Krupka $^{\mathrm{a}, \mathrm{c}, \mathrm{d}}$, S. Singh ${ }^{\mathrm{d}, \mathrm{c}}$, D. Neely ${ }^{\mathrm{e}}$, \\ V. Olšovcová ${ }^{b}$ and D. Margarone ${ }^{\mathrm{f}, \mathrm{b}}$ \\ ${ }^{a}$ Faculty of Nuclear Sciences and Physical Engineering, Czech Technical University in Prague, Czech \\ Republic \\ ${ }^{b}$ Institute of Physics ASCR, v.v.i (FZU), ELI-Beamlines, Prague, Czech Republic \\ ${ }^{c}$ Institute of Physics of the Czech Academy of Sciences, Prague, Czech Republic \\ ${ }^{d}$ Institute of Plasma Physics of the Czech Academy of Sciences, Prague, Czech Republic \\ ${ }^{e}$ Central Laser Facility, STFC Rutherford Appleton Laboratory, United Kingdom \\ ${ }^{f}$ Centre for Plasma Physics, School of Mathematics and Physics, Queen's University Belfast, Belfast, \\ United Kingdom \\ E-mail: Valeriia.Istokskaia@eli-beams.eu
}

ABSTRACT: With the development of high-intensity and high-repetition rate laser systems, it has become crucial to be able to detect and characterize in real time the high-energy byproducts (mainly electrons and photons) of laser-generated plasma. A novel multi-purpose scintillatorbased electromagnetic calorimeter focused on high-energy particle and photon measurements and capable of working on a shot-by-shot basis at high-repetition rate is being developed at the ELI Beamlines center. Preliminary tests of this device under photon and electron irradiation from conventional and laser-driven sources are summarized and the results are here presented. A corresponding signal unfolding technique which was ad-hoc developed to reconstruct energies of one or two thermal populations in short time is described in detail.

KEYWORDS: Scintillators; Radiation and particle detection; Signal unfolding; Gamma rays, High-energy X-rays, Laser-plasma characterization

\footnotetext{
* Corresponding author.
} 


\section{Contents}

1. Introduction 1

2. Design 2

3. Signal unfolding technique 4

4. Preliminary experimental tests and verification of the unfolding technique 5

4.1 Comparative study of the photon and electron energy deposition along the device 6

$\begin{array}{ll}4.2 \text { Experimental tests in laser-plasma environment } & 7\end{array}$

$\begin{array}{ll}\text { 4.2.1 Electron measurement at TERESA } & 7\end{array}$

$\begin{array}{lr}\text { 4.2.2 Photon measurement at PALS } & 9\end{array}$

5. Further development and prospective 10

6. Conclusions $\quad 12$

\section{Introduction}

The interaction of a petawatt (PW)-class laser pulse with matter induces the ionization of the target material, resulting in instantaneous plasma creation and the subsequent emission of a copious amount of energetic particles and photons $[1,2]$. Typically, the laser energy is rapidly transferred to a population of relativistic electrons, often referred to as "hot electrons" $[3,4,5]$. While propagating through the plasma, such electrons emit mainly Bremsstrahlung radiation which falls within the $\mathrm{X} / \gamma$ ray region of the electromagnetic spectrum $[6,7]$. Such energetic photon emission is of great interest both for fundamental and applied research since it can be used, inter alia, for the investigation of the electron temperatures and electron transport during laser-plasma interaction $[8,9]$, the induced proton and ion beam production characterization $[10,11]$, radiography of dense objects [12, 13], radiation chemistry [14], and cancer radiotherapy [15]. Moreover, compared to conventional X/ $\gamma$-ray sources, laser-driven systems can offer a wide range of beam parameters (energy, flux) suitable for a particular user by modifying laser or target conditions [16].

However, high energy $(>\mathrm{MeV})$ and short duration (fs) of the radiation generated from the laser-plasma interaction $[17,18]$ make the detection significantly challenging. Over the past years, multiple photon detectors have been developed and employed; various spectrometers based on differential filtering [19], Compton scattering [20,21] and stack of absorbing materials [22] have proved to be among the most advantageous. The common sensitive part of such spectrometers is an image plate (usually FUJI-film [23, 24]) characterized with high sensitivity to radiation and resistance to the electromagnetic pulse (EMP), which accompanies each interaction and can disrupt electronic equipment [25].

However, after each shot an image plate needs to be dismounted and subjected to a scanning process by a specialized reader, making the post-processing of an image-plate-based 
detector highly time-consuming and thus limiting the experimental repetition rate. Moreover, the output depends dramatically on the scanning conditions, hence the comparative study of different shots may be problematic [26].

State-of-the-art laser facilities aim at a high-repetition rate working regime, as well as at high laser intensities, e.g. up to $10 \mathrm{~Hz}$ and $10^{22} \mathrm{~W} / \mathrm{cm}^{2}$ for the $1 \mathrm{PW}$ HAPLS laser at ELI Beamlines [27]. Therefore, a novel approach for the radiation detection is required. A new type of diagnostics should enable real-time readout and data processing with respect to the high shot frequency. Furthermore, it should provide an accurate spectral information about the highintensity induced energetic radiation which can be compared shot-to-shot.

As has been recently demonstrated in [28], the usage of scintillators represents an alternative approach to measuring X-rays from the laser-plasma interaction. Such materials emit visible light as a result of internal electrons de-excitation after being exposed to the incoming radiation or particles. The duration of such emission varies from hundreds of nanoseconds to hundreds of picoseconds depending on the material, which is below the expected repetition rates at the current and forthcoming laser systems.

Being assembled in an array, scintillating crystals form an electromagnetic calorimeter, acting as absorbing and detecting media at the same time: electromagnetic cascades are generated as particles deposit their energy along the crystals. The scintillation light produced corresponds to the particles temperature and hence energy distribution.

Scintillating materials are not affected by the EMP. Moreover, an optical signal readout (implemented e.g. via CCD or CMOS camera) can be chosen to reduce the EMP-induced electrical noise while converting the scintillation light into electrical form. Additionally, advantages of the scintillator-based detector include its portability and cost-effectiveness owing to its compact size, provided that the density of a scintillator is high enough for the radiation of a particular energy.

Hereafter, we will describe a novel design of a scintillator-based electromagnetic calorimeter for measuring high-energy radiation from laser-plasmas in the high-repetition rate regime, primarily focused on the $\mathrm{MeV}$ range for $\mathrm{PW}$-class lasers. The unfolding technique specifically developed for the calorimeter signal reconstruction will be described in detail, along with preliminary experimental tests. Moreover, it will be anticipated that the device, primarily focused on plasma radiation characterization, has the capacity to be a flexible and effective tool for multi-purpose measurements, including electron and ion detection, without significant changes in the setup and signal post-processing.

\section{Design}

According to the literature studies [29, 30], Bremsstrahlung emission seems to be the dominant radiation process generated in laser-plasma interaction for laser intensities below $10^{22} \mathrm{~W} / \mathrm{cm}^{2}$; for higher intensities, nonlinear Compton scattering is likely to prevail [31]. It has been experimentally observed that such Bremsstrahlung spectrum may consist of at least two Maxwell-Boltzmann (MB) energy distributions [32, 33] caused by separate populations of accelerated electrons. Therefore, for our calorimeter design it was decided to use two different scintillating materials in an array-like setup, in order to disentangle and measure the high and low-energy components of the photon spectrum simultaneously.

A diagram of the scintillator-based electromagnetic calorimeter is shown in Fig.1.The frontal part of the device was chosen to be composed of plastic (EJ-200) scintillators, lowdensity $\left(1.03 \mathrm{~g} / \mathrm{cm}^{3}\right)$ organic crystals with a high light yield $(10000$ photons $/ \mathrm{MeV})$. Such 
crystals are supposed to resolve the low-energy component of the incident radiation, barely affecting the high-energy one. The second part of the detector is constituted by inorganic high- $Z$ Bismuth Germinate (BGO) scintillators, characterized by high density $\left(7.13 \mathrm{~g} / \mathrm{cm}^{3}\right)$ and hence capable of effective interaction with the remaining energetic photons (light yield is 8000 photons $/ \mathrm{MeV}$ ). The chosen scintillating materials are commercially available and cost-effective.

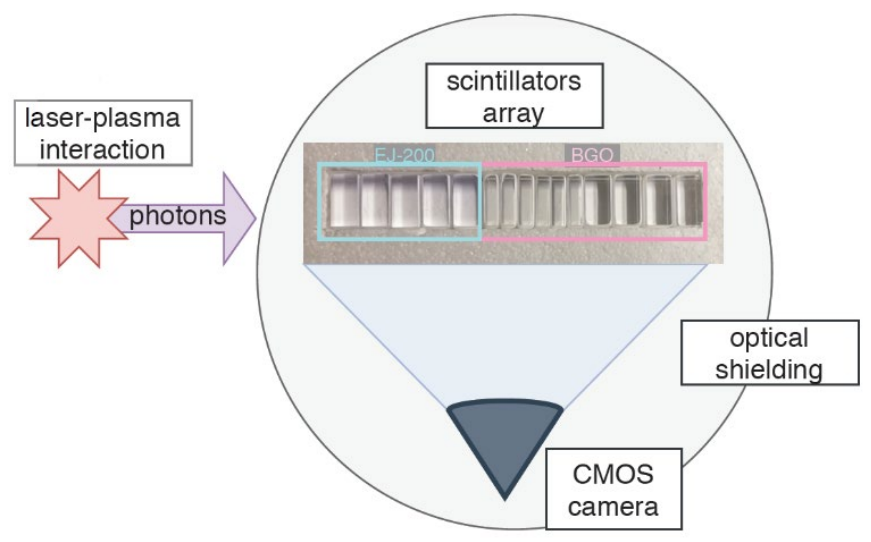

Figure 1. Sketch of the developed scintillator-based electromagnetic calorimeter: two scintillating materials (EJ-200 and BGO) are placed in a holder, the optical signal from the crystals is recorded by a CMOS camera. The crystals and the camera are put inside a black covering to shield the device from the external optical light. The whole setup is exposed to the radiation from the laser-plasma interaction.

Each crystal is wrapped in white polytetrafluoroethylene (PTFE) tape leaving one side uncovered for the light detection. Usage of the PTFE improves the uniformity of the scintillation light, enhances the signal-to-noise ratio, and also minimizes the light transmission between adjacent crystals. Since the PTFE wrapping is around $0.7 \mathrm{~mm}$, the attenuation of energetic radiation is small [34] and can be neglected.

The total length of the sole crystal array is $145 \mathrm{~mm}$, complying with the requirements of compactness and portability of the device. The array needs to be placed in a holder (i.e. a polystyrene box with specially carved space or an adjusted 3D-printed plastic box) which usually adds a 10-20 $\mathrm{mm}$ to its total length, depending on the design. The design can be adapted for the needs of an experiment by adding or reducing the number of crystals and hence modifying the length. Since the highest energy deposition is anticipated at the region where radiation hits the densest material (i.e. in the first BGO crystals), the first six BGOs were decided to be half the length of the other crystals $\left(20 \times 5 \times 20 \mathrm{~mm}^{3}\right.$ versus $\left.20 \times 10 \times 20 \mathrm{~mm}^{3}\right)$ to ensure better energy resolution at the peak region.

The choice of materials and dimensions of the scintillating crystals were subject of a specific study based on the Monte Carlo simulations performed in FLUKA [35, 36, 37]. The calorimeter, the holder and PTFE wrapping were modelled using the Flair software [38]. The interaction of photons with the detector was studied, with photons following the MB distribution with a characteristic temperature ranging from $10 \mathrm{keV}$ to $40 \mathrm{MeV}$. The upper bound of the simulation range is correlating with the hot electrons temperature estimated for the laser intensities around $10^{22} \mathrm{~W} / \mathrm{cm}^{2}$ according to the scaling laws described in [39, 40]. The assumption is taken that the maximum temperature of photons is equal or less than that of the hot electrons. The details of this study will be described in a dedicated paper. 
The read-out of the light signal was represented by a CMOS Manta-235G camera coupled with an $8.5 \mathrm{~mm}$ focal length objective. The camera's maximum frame rate at full resolution is $50.8 \mathrm{fps}$, while the decay time for EJ-200 and BGO is $2.1 \mathrm{~ns}$ and $300 \mathrm{~ns}$, respectively. Coupled with the camera, the device can achieve the maximum repetition rate of $50 \mathrm{~Hz}$, which is well beyond the abilities of the current and near-future laser systems where the calorimeter is planned to be used (e.g. $10 \mathrm{~Hz}$ for the HAPLS laser).

The setup including the detector (scintillators inside a holder) and the camera is properly covered with a black foil to ensure a proper shielding from external optical light.

\section{Signal unfolding technique}

In order to proceed from the experimentally measured calorimeter signal to the physical quantities, such as photons energy distribution, a method for signal reconstruction has been developed.

Based on the performed Monte Carlo simulations, it is possible to create a so called response matrix $R \in \mathbb{R}^{m \times k}$, where $m$ is the number of crystals in which energy deposition was scored and $k$ is the number of the simulated temperatures/energies. A particular response matrix depends on the given detector design, the type of radiation to be detected, and temperature/energy range. The matrix points are interpolated using cubic splines, making the matrix continuous to improve the unfolding accuracy.

The measured signal $S$ is considered to be a combination of two photon groups of different temperature $\left(T_{1}, T_{2}\right)$ and "number of particles" $\left(N_{1}, N_{2}\right)$. Nevertheless, since the real number of particle cannot be determined with this method, $N_{1}$ and $N_{2}$ can only provide us with a relative representation of the two population.

Given that $f\left(i, T_{l}\right)$ is the interpolation function of the response matrix, the signal at the $i$-th crystal can be written in a vector form as:

$$
S_{i} \equiv N_{1} \cdot f\left(i, T_{1}\right)+N_{2} \cdot f\left(i, T_{2}\right) .
$$

Let $\bar{S}$ be the "guessed" response as a result of the unfolding:

$$
\bar{S}_{i} \equiv \bar{N}_{1} \cdot f\left(i, \bar{T}_{1}\right)+\bar{N}_{2} \cdot f\left(i, \bar{T}_{2}\right) .
$$

The difference between the "true" and the "guessed" detector response is given by the $\chi^{2}$ function which approaches zero if the guess $\left(\bar{N}_{1}, \bar{T}_{1}, \bar{N}_{2}, \bar{T}_{2}\right)$ is correct:

$$
\chi^{2}\left(\overline{N_{1}}, \overline{T_{1}}, \overline{N_{2}}, \overline{T_{2}}\right)=\sum_{i=1}^{m}\left(S_{i}-\bar{S}_{i}\right)^{2} .
$$

Thus, the goal of the unfolding procedure is to determine the four parameters $\left(N_{1}, T_{1}, N_{2}, T_{2}\right)$ by minimizing the $\chi^{2}$ function.

For this purpose, two consecutive numerical algorithms have been developed, forming an unfolding technique. The first one is a standard method based on the signal comparison with a number of simulated signals $\overline{S_{n}}$, using a large step between the parameters $\left(\bar{N}_{1}, \bar{T}_{1}, \bar{N}_{2}, \bar{T}_{2}\right)$. The parameters are investigated inside wide intervals determined according to the expectations for a particular type of experiments. Usually, intervals cover a wide range of values: in our case, it is $10 \mathrm{keV}-40 \mathrm{MeV}$ for $\left(\bar{T}_{1}, \bar{T}_{2}\right)$, given by the generated response matrix; while the $\left(\bar{N}_{1}, \bar{N}_{2}\right)$ interval is connected with amplitudes of the response matrix and the signal and covers three to 
four orders of magnitude. The first step is supposed to provide with the first rough guess, which is then used as a starting vector in the second algorithm. The latter one is called the gradient unfolding algorithm, as it calculates the gradients of the $\chi^{2}$ function, allowing to determine the direction of the function's strongest decrease and subsequently move the starting vector in this direction, approaching the minimum. The gradient unfolding uses a nonlinear regression model (implemented via fitnlm function in Matlab) in order to obtain the standard error of each parameter, along with various statistical parameters such as p-value and adjusted R-squared [41]. Such information enables to interpret whether the unfolding output is trustable or not.

The precision of the technique depends strongly on the step size and the intervals' width in the first algorithm which influences the first guess. The reason is that depending on the input the second algorithm may terminate in one of the local minima of the $\chi^{2}$ function instead of the global one of the interest. Unfortunately, it is not possible to determine which type of minima has been found, since the numerical analysis, on which the unfolding algorithm is based, does not allow such an option. Nevertheless, by choosing appropriate intervals for the parameters $\left(\bar{N}_{1}, \bar{T}_{1}, \bar{N}_{2}, \bar{T}_{2}\right)$ and using a smaller step in the first part of the algorithm, it is possible to reduce the probability of finding a local minimum instead of a global one. However, a compromise between computational time and precision needs to be defined depending on the analysis requirements.

The unfolding technique described above can be applied also to reconstruct 1-T signal, reducing the number or the function parameters from four to one $\left(T_{1}\right)$. Moreover, it is possible to extrapolate the technique aiming for more than 2-T unfolding, if needed. However, the increased number of parameters may be computationally demanding to evaluate and more sensitive to local minima. It is also worth noting that the technique is not limited only to the MB distributed radiation but can be used also e.g. for a monoenergetic case (provided that the corresponding response matrix is created).

The execution time for the 2-T unfolding was usually between 100 and $200 \mathrm{~s}$, depending on the particular signal, while for the 1-T unfolding was around $10 \mathrm{~s}$. However, the algorithm was run on an ordinary COTS (Commercial Off-The-Shelf) computer. By using more powerful systems or a dedicated machine it is possible to decrease the computational time.

\section{Preliminary experimental tests and verification of the unfolding technique}

The first prototype of such scintillator-based electromagnetic calorimeter has been designed and constructed at ELI Beamlines in the intention to use it during the upcoming commissioning experiments using the HAPLS PW-class laser system available at the ELIMAIA beamline (ELI Multipurpose Applications of Ion Acceleration) [42]. The calorimeter is expected to provide online shot-to-shot Bremsstrahlung measurements and to determine the hot electrons temperature which is of high importance for the studies of the laser-plasma interaction and laser-driven ion acceleration planned at ELIMAIA.

However, as it will be demonstrated and discussed in the following, besides Bremsstrahlung radiation, the calorimeter setup and the unfolding principle allow to use it for the detection of various kinds of incoming particles, including electrons and protons. Furthermore, the possibility of having a multi-purpose portable device can be beneficial in the laser-plasma experiments, where a wide range of particles is produced.

To demonstrate the efficacy and flexibility of the current detector design, a few proof-ofprinciple tests were carried out at different experimental facilities, including conventional 
(Microtron MT25) and laser-driven radiation sources (PALS and TERESA), during which the detector was exposed to energetic photon and electron beams.

\subsection{Comparative study of the photon and electron energy deposition along the device}

Before usage in laser-plasma environment, the calorimeter and the unfolding technique were preliminary tested at the microtron MT25 of the Nuclear Physics Institute of the Czech Academy of Sciences, a conventional circular electron accelerator with an energy range between 6 and $25 \mathrm{MeV}$ and a maximum current of $20 \mathrm{~mA}$ [43]. This experimental run allowed not only to perform a proof-of-principle demonstration under real experimental conditions but also to compare signal characteristics while measuring photons or electrons and comprehend the difference in the energy deposition behavior between these two kinds of radiation.

The detector setup and crystal configuration were following the scheme described in Fig.1. Additionally, a $5 \mathrm{~cm}$ thick $\mathrm{Pb}$ box was used to shield the detector and the camera from secondary/external radiation. The radiation was propagating inwards through a $3 \mathrm{~mm}$ collimator. The detector was aligned perpendicularly to the collimator along its axis. The distance between the collimator and the microtron exit was $150 \mathrm{~cm}$. Accelerated electrons traveled along it in air.

Two different kinds of measurements were performed using electrons accelerated to the energy of $16 \mathrm{MeV}$. After the passage in air, electrons were attenuated down to approximately 15.6 MeV. In the first measurement, a continuous electron beam was sent directly to the calorimeter in order to measure monoenergetic electrons. In the second one, a Tungsten convertor and an $\mathrm{Al}$ electron stopper were placed on the electron path, enabling to generate Bremsstrahlung radiation and simultaneously cut the electrons in order to measure $\mathrm{MB}$ distributed photons.

The raw and processed signals for both measurements are presented in Fig. 2 a) and b). The beam is incident from the left side. As can be seen, electrons interact mainly with the light plastic part of the detector causing it to scintillate, being completely stopped in the first BGO crystal due to its high density. On the other hand, photons demonstrate opposite deposition behavior due to their different fundamental characteristics: they interact only weakly with the low-density EJ-200, having the maximum deposition in the BGO part. Such difference in the energy deposition behavior of electrons and photons facilitates a correct identification of the incoming radiation source. It can also provide with an opportunity to measure and characterize their blended emission simultaneously using a two-material scintillator array, which can be useful when multiple kinds of particles are considered.
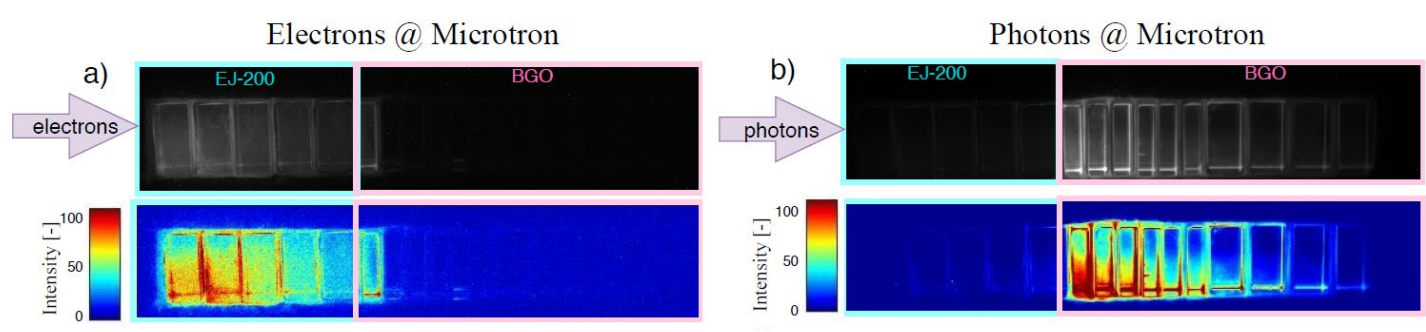

Figure 2. Comparison between the detector response when it is exposed to the Microtron accelerated electrons (a) and to the secondary Bremsstrahlung photons (b). The top figures show the raw signal from the camera and the bottom ones the post-processed signal. 


\subsection{Experimental tests in laser-plasma environment}

The preliminary test described in the previous session represented a proof-of-principle demonstration of the electromagnetic calorimeter under continuous irradiation. The following step for validation of the newly developed diagnostic consisted in its irradiation by laser-plasma byproducts and subsequent tests of the unfolding. The laser-plasma environment is usually considered harsh for active detectors due to ultrashort and ultra-intense plasma radiation emission. Both electrons and photons were measured separately by the calorimeter in two different experimental campaigns conducted at two laser facilities, where the laser intensity and energy ranges were also different.

\subsubsection{Electron measurement at TERESA}

TERESA (TEstbed for high REpetition-rate Sources of Accelerated particles) is a laserplasma target area available at ELI Beamlines, mainly used to test and develop innovative high repetition-rate targetry and diagnostic systems for laser-plasma characterization [44]. TERESA uses a sub-aperture of the Ti:Sapphire HAPLS laser system [45] with the following parameters: $1.3 \mathrm{~J}$ maximum pulse energy, 30 -fs pulse duration (FWHM), and up to $10-\mathrm{Hz}$ repetition rate. The peak power on target exceeds $30 \mathrm{TW}$ and the laser pulse can be focused down to reach laser intensity in the range $10^{19}-10^{20} \mathrm{~W} / \mathrm{cm}^{2}$.

The calorimeter was located in vacuum inside the interaction chamber, at the front side of the laser-target interaction and aligned along $-15^{\circ}$ with respect to the target normal. A shorter version of the detector was used to fit inside the chamber: the number of EJ-200 crystals was reduced from five to three, and the last three BGO crystals were removed. The Manta camera was installed outside the chamber, looking at the detector through a glass viewport. Since no optical shielding was implementable in this case, the following approaches were applied: i) an optical bandpass filter $(460 \pm 10) \mathrm{nm}$ was mounted on the camera, enabling to cut the first and the second laser harmonics $(820 \mathrm{~nm}$ and $410 \mathrm{~nm}$, resp.) and at the same time to transmit the scintillation light; ii) the experimental area was lightened down. The camera was synchronized with the laser: during each shot, it was being triggered $50 \mathrm{~ms}$ before the main pulse, recording for $100 \mathrm{~ms}$, and then saving the signal in an automatic mode.

During the measurements, $1.3 \mathrm{~J}$ of the laser energy was delivered onto a $23 \mu \mathrm{m}$ thick polyethylene plastic target using a $15^{\circ}$ laser incidence angle and about $10^{19} \mathrm{~W} / \mathrm{cm}^{2}$ pulse intensity.

The vacuum position of the scintillator array enabled detecting hot electrons emitted from the laser-plasma interaction, otherwise the chamber walls could absorb part of the electrons. In these specific irradiation conditions, the average flux of the Bremsstrahlung radiation was below the detection threshold of the calorimeter since the setup was not optimal for maximizing the gamma-ray signal (low atomic number target element and low laser energy). In fact, simultaneous measurements recorded by another calorimeter placed outside the vacuum chamber (in air), did not detect any useful signal. Furthermore, to avoid possible ion capturing, a black aluminum foil ( $50 \mu \mathrm{m}$ thick) was placed on the front side of the calorimeter.

The signal measured by the calorimeter placed in vacuum is shown in Fig. 3 (top). As can be seen, the maximum amount of energy was deposited in the first EJ-200 crystal. Such deposition behavior, involving mainly the plastic calorimeter part, is typical for electrons, as it was shown in the previous section. Moreover, the expected energy range for the given laser parameters allowed to exclude any dominance of high flux low-energy Bremsstrahlung photons 
(tens of eV) that could potentially generate a similar energy deposition, hence signal on the detector. Therefore, only electrons were considered during the signal reconstruction.

During the unfolding process, only the first four crystals were taken into account (3 plastic and the first BGO). The reason is the low signal-to-noise ratio and the glass flange induced light reflections in the rest of the stack. It is worth noting that considering the plastic-BGO border is important to eliminate the case of photons in the unfolding (as it corresponds to the region of a significant energy deposition for photons). During the 2-T signal reconstruction, the model considered in the unfolding technique demonstrated statistical irrelevance of the second temperature for this case. Most probably, the second (cold) population of electrons was cut by the aluminum foil located at the front side of the device (for the given thickness, electrons below $60 \mathrm{keV}$ are stopped within the foil). The unfolded result for $1-\mathrm{T}$ is $(964.0 \pm 57.1) \mathrm{keV}$, with R-Squared $=0.999$ and $\mathrm{p}$-value $=4 \times 10^{-4}$. It is worth emphasizing that the uncertainty of the temperature found accounts only for the mathematical uncertainty of the unfolding procedure. A dedicated study of the systematic uncertainty of the algorithm is ongoing. The comparison between the measured (blue line) and the unfolded signal (orange line) is demonstrated in Fig. 3 on the bottom. The uncertainty of the experimental data is given by the standard mean error (SME) for a given region of interest (ROI) - the crystal area. Given the huge number of pixels in each ROI, the SME is small for each crystal; therefore, we neglect it in the data processing (unfolding).

According to the ponderomotive scaling laws derived in [46, 47], suitable for the given laser conditions, the temperature of the hot electrons for the given parameters of the short-pulse laser was estimated to be in the range $750-1000 \mathrm{keV}$. The unfolding result falls within the expected range. However, for the laser intensities beyond $10^{19} \mathrm{~W} / \mathrm{cm}^{2}$ there are still few experimental data available to clarify the scaling. The correlations between the detector's measurements and the scaling laws might be studied in future.

A proof-of-principle for a repetition-rate working regime was also demonstrated by the calorimeter during its tests at TERESA. The maximum achievable shot frequency, in terms of detector data acquisition and processing, during the experiment was approximately $0.03 \mathrm{~Hz}$ (two shots per minute).

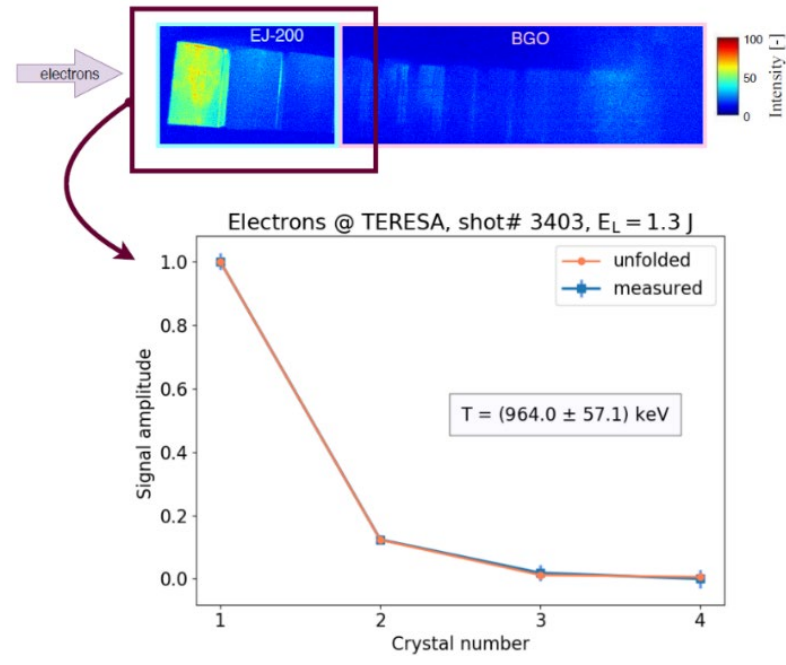

Figure 3. Top: The scintillating detector after being exposed to the hot electrons inside the chamber (processed image). A plastic target of $23 \mu \mathrm{m}$ was used. Bottom: the comparison between the measured and the reconstructed signal using the unfolding algorithm. The measured points are given by the average signal intensity in each crystal. 


\subsubsection{Photon measurement at PALS}

An experimental measurement of photons generated in laser-plasma via the Bremsstrahlung process was carried out at the Prague Asterix Laser System (PALS) facility [48], where an iodine laser was available and operated in a single-pulse regime at $1315 \mathrm{~nm}$ and delivered a maximum laser energy of $620 \mathrm{~J}$ in about $350 \mathrm{ps}$. The laser was focused down to moderate laser intensities (slightly above $10^{16} \mathrm{~W} / \mathrm{cm} 2$ ) onto various solid targets (metals and plastic), having the focal spot size around $100 \mu \mathrm{m}$.

The number, type, and thickness of crystals were the same as described in the Design section. Additionally, the calorimeter setup in this case included a plastic crystal holder developed to ensure better mounting capability and to optically separate the crystals from each other. The detector was placed outside the vacuum chamber in front of the glass viewport at the distance of $83.7 \mathrm{~cm}$ from the target and aligned along the target normal. The whole setup (detector and a camera) had $5 \mathrm{~cm}$ thick $\mathrm{Pb}$ shielding on the front side for the protection from unwanted secondary radiation, leaving a $1.5 \times 1.5 \times 5 \mathrm{~cm}^{3}$ hole in front of the detector. The experimental setup is sketched in Fig. 4.

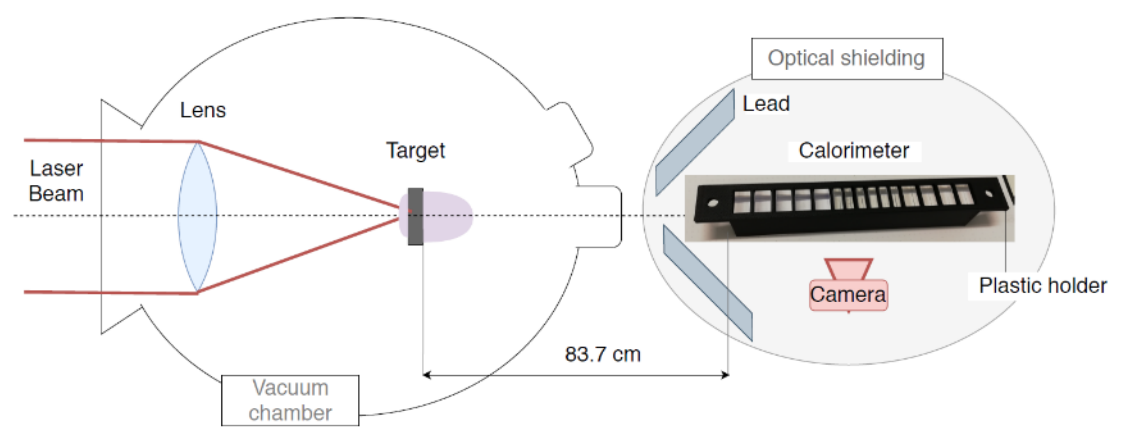

Figure 4. Experimental setup during the PALS experiment: the calorimeter is located outside of the vacuum interaction chamber at the target normal axis.

During the experiment, metals of various thickness and atomic number were tested as a target material. The calorimeter signal for the case of the $10 \mu \mathrm{m}$ thick tantalum (Ta) target is shown on the top of in Fig. 5. According to [6, 49], Ta is considered as an efficient bremsstrahlung converter due to its high $\mathrm{Z}$. As can be seen, the strongest signal is recorded in the first BGO crystal: the measured photons were interacting weakly with the light plastic part, depositing almost all of the energy once they reached the denser region. However, the radiation energy combined with the flux were not sufficient to contribute a strong signal in the remaining crystals.

The comparison between the measured signal (blue line) and the unfolding result (orange line) is shown on the bottom side of Fig. 5. The error bars are calculated as SME for each crystal area, similarly as in the abovementioned campaign. During the signal reconstruction, the very last 3 crystals were not taken into consideration due to the low signal and low signal-tonoise ratio. The unfolding technique was not able to determine more than one non-zero temperature, although two MB distributed photons populations were expected. The model 
evaluated the second component to be statistically insignificant, suggesting that no second temperature was present. Most probably, the temperature of the second photons population was below the detection limit $(10 \mathrm{keV})$ in this specific setup. The unfolded MB temperature of the photons is $(60.6 \pm 2.6) \mathrm{keV}$, the R-squared is 0.999 , and $\mathrm{p}$-value is $10^{-10}$. The result is in the same range as the hot electrons temperatures $(50-100 \mathrm{keV})$ estimated by electron spectrometers used in the previous experiments at PALS under similar experimental conditions $[52,51]$, which is in accordance with the expectations.
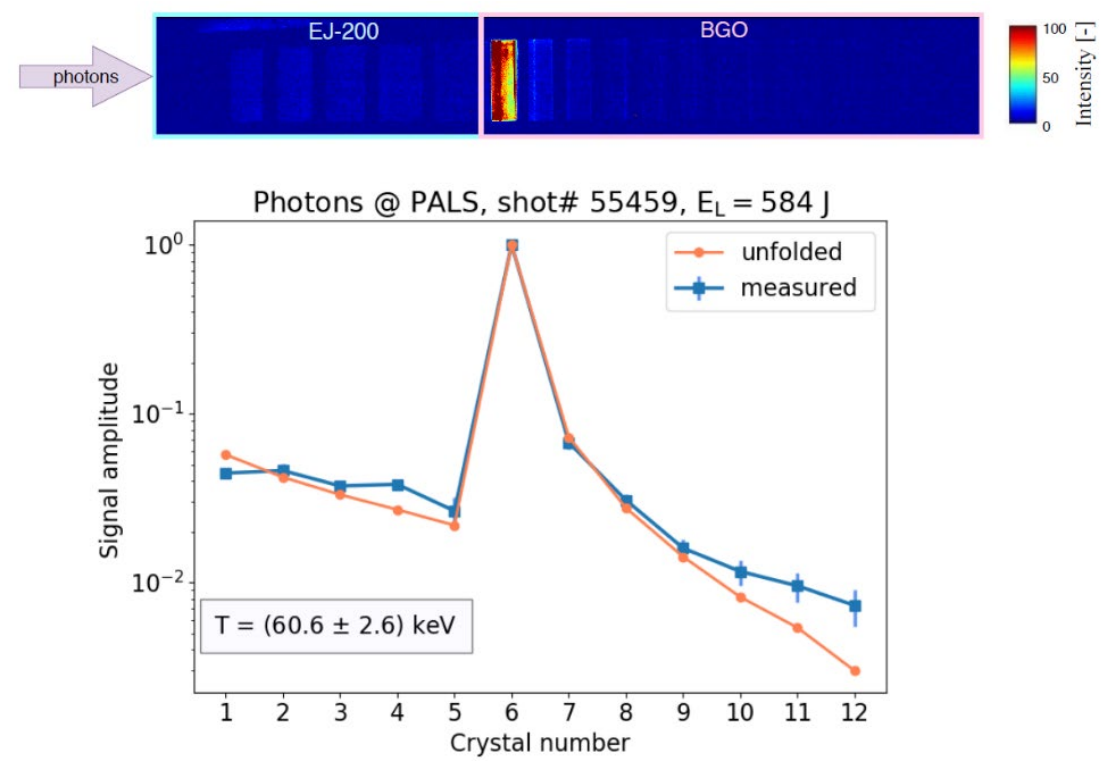

Figure 5. Top: the scintillating detector after being exposed to the X-ray radiation coming from the laser-plasma interaction. A Ta target of $10 \mu \mathrm{m}$ was used .The majority of the photons energy is deposited in the first BGO crystal. Bottom: The comparison between the measured signal and the unfolding algorithm output. The measured points are given by the average signal intensity in each crystal.

\section{Further development and prospective}

Despite being designed for high-energy (tens of $\mathrm{MeV}$ ) and high-repetition rate measurements of radiation at laser-plasma experiments, the electromagnetic calorimeter has not been in a position yet to be applied under proposed conditions at the state-of-the-art laser facilities and demonstrate its full capacity. Such experiments are planned in the near future. Nevertheless, the device has proved to be trustable near its lower detection limit during the tests using tens/hundreds of $\mathrm{keV}$ irradiation, as regards both electrons and photons (X-rays). However, to be used preferentially in that energy range, some modifications can be applied on the design to achieve better resolution: in particular, reduction of the crystals thickness, adjustment of the crystals number, and usage of smaller density types of scintillators. A more sensitive EMCCD camera might be also used to record the low-intensity scintillation light in this case.

Among the most essential steps is the calibration of the detector, including calibration in terms of energy, distance and angle of the crystal position in the holder in relation to the camera, and crystal by crystal fluctuations in light output. For lower energies calibration (up to $\sim 1.5$ $\mathrm{MeV}$ ) natural sources of radiation like Co or Cs were used, while for higher ones (tens of $\mathrm{MeV}$ ), the usage of conventional particle accelerators or medical radiation sources is planned. The 
calibration studies and corresponding tests are a subject matter of a separate publication currently in preparation.

As described above, the unfolding technique is based on the response matrix resulting from Monte Carlo simulations of the radiation energy deposition inside the calorimeter. Better accuracy of the unfolding may be achieved by increasing the number of matrix points and/or the statistics of each of such points. Another approach is simulating and following directly the scintillation light expansion instead of the deposited energy. The possibilities mentioned should be studied with regard to the time consumption and accuracy. Also, further optimization of the algorithm can be achieved to reduce the unfolding time.

Another prospective is the employment of the calorimeter for simultaneous detection of Xrays and electrons generated in the laser-plasma interaction. Due to the difference in the energy deposition in the two-part calorimeter demonstrated above, it may be possible to distinguish and characterize such radiation. However, specific simulations and subsequent tests in an experiment (including vacuum location of the device for electron irradiation and usage of the high- $Z$ target for the strong Bremsstrahlung generation) will be required in this case.

Apart from photons and electrons, similar design of the electromagnetic calorimeter can theoretically be used also for high-energetic protons measurements. Protons accelerated up to $300 \mathrm{MeV}$ by the laser-plasma interaction are anticipated at the ELIMAIA beamline [34]. Application fields of such protons will include, inter alia, medical research for tumor treatment and cultural heritage studies. For such purposes, a low-divergent narrow proton beam is used which should be properly characterized. As was described in [52], scintillators proved to be suitable for $\sim 10 \mathrm{MeV}$ proton beams measurements. We performed FLUKA simulations to study the energy deposition behavior of a pencil-like proton beam in the calorimeter developed. As can be seen in Fig. 6, the detector composition and dimensions are suitable for high-energy proton irradiation expected at ELIMAIA and fully contain the Bragg peak: the case for the 60 $\mathrm{MeV}$ protons of which is usually used for ocular proton therapy [53] is demonstrated on the top, while the maximum expected proton energy case is shown on the bottom.

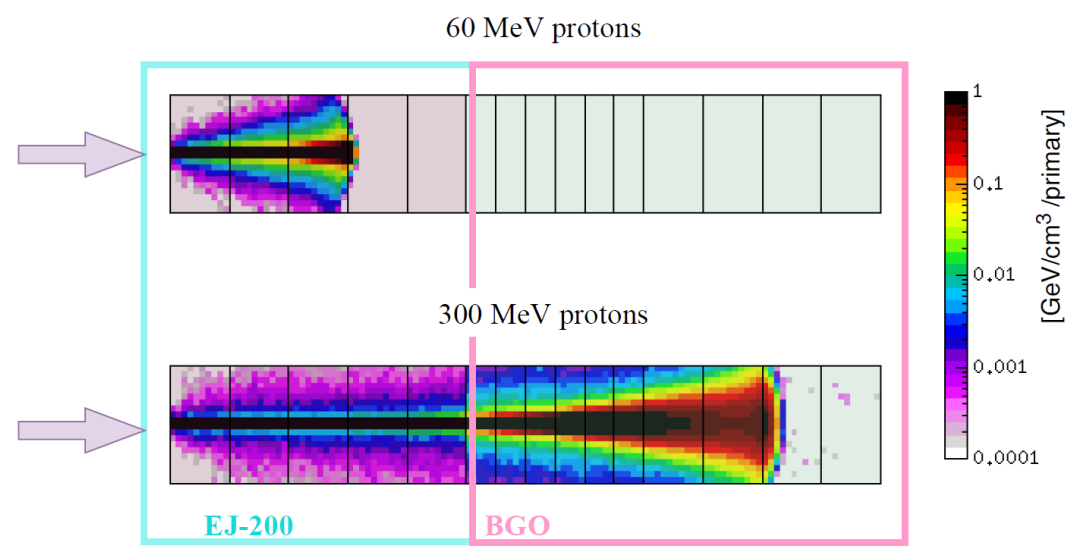

Figure 6. The energy deposition of protons in the electromagnetic calorimeter in the case of $60 \mathrm{MeV}$ (top) and $300 \mathrm{MeV}$ (bottom). 


\section{Conclusions}

The preliminary tests of a novel scintillator-based electromagnetic calorimeter together with a corresponding signal unfolding technique were carried out in a laser-plasma environment. A comparative study of the photon and electron energy deposition along the device was performed at the microtron, a conventional circular electron accelerator. Versatility and reliability of the device when exposed to irradiation of different kinds of particles (electrons and photons) were experimentally demonstrated at two laser facilities offering different laser parameters (intensity, energy, etc.) in the Czech Republic (TERESA target area at ELI Beamlines and the PALS laser facility).

The calorimeter is characterized with wide range of detectable energies: during the first tests, the device has proved to be operational both for low (tens of $\mathrm{keV}$ ) and high (tens of $\mathrm{MeV}$ ) temperatures of the incoming radiation. However, it is primarily focused on working in highenergy region (up to $\mathrm{T}=40 \mathrm{MeV}$ for photons). The detection range can be tuned, if necessary, by, inter alia, changing the crystals thickness and/or exchanging the camera for a more sensitive one (e.g. EMCCD).

The possibility to achieve a high-repetition rate working regime is given by the scintillators decay time and the readout camera parameters, making the device a promising tool for the shotto-shot measurements. Optical signal readout allows to reduce the effects of the EMP during laser-plasma experiments.

A special signal unfolding technique was developed to reconstruct energies of one or two (possible to expand for more) thermal population of radiation. The execution time is $\sim 10 \mathrm{~s}$ for single and $\sim 200 \mathrm{~s}$ for double temperature unfolding (for an ordinary computer), enabling to perform data analyses in a short time during experiments.

Calibration of the overall system, optimization of the signal unfolding technique, and the corresponding simulation studies are among the most significant aspects that are currently being investigated.

Prospective use for different radiation detection, e.g. ions or a mixture of electrons and photons, were also discussed herein, making the calorimeter a promising candidate for the multi-purpose applications.

\section{Acknowledgments}

This work has been supported by the Ministry of Education, Youth and Sports of the Czech Republic (Project No. LQ1606, No. LM2015083 (PALS RI), and No. EF16_013/0001552), by the project "Advanced Research Using High Intensity Laser Produced Photons and Particles" (CZ.02.1.01/0.0/0.0/16_019/0000789), by the Access to the PALS RI under the EU LASERLAB IV project (Grant Agreement No. 654148), and by the Czech Science Foundation (Grant Nos. 19-02545S). The authors gratefully acknowledge the support of the PALS and the ELI Beamlines technical staff.

\section{References}

[1] T. Nakamura, et al., "High-Power $\gamma$-Ray Flash Generation in Ultraintense Laser-Plasma Interactions," Phys. Rev. Lett. 108 (2012) 195001

[https://doi.org/10.1103/PhysRevLett.108.195001] 
[2] C. P. Ridgers, et al., Dense Electron-Positron Plasmas and Ultraintense $\gamma$ rays from Laser-Irradiated Solids, Phys. Rev. Lett. 108 (2012) 165006

$$
\text { [https://doi.org/10.1103/PhysRevLett.108.165006] }
$$

[3] S. C. Wilks, et al., Absorption of ultra-intense laser pulses, Phys. Rev. Lett. 69 (1992) 1383

[https://doi.org/10.1103/PhysRevLett.69.1383]

[4] M. Sherlock, Universal scaling of the electron distribution function in one dimensional simulations of relativistic laser-plasma interactions, Physics of Plasmas 16.10 (2009) 103101

[https://doi.org/10.1063/1.3240341]

[5] M. G. Haines, et al., Hot-electron temperature and laser-light absorption in fast ignition, Phys. Rev. Lett. $102(2009) 045008$

[https://doi.org/10.1103/PhysRevLett.102.045008]

[6] J. Galy, et al., Bremsstrahlung production with high-intensity laser matter interactions and applications, New journal of Physics $\mathbf{9 . 2}$ (2007) 23

[https://doi.org/10.1088/1367-2630/9/2/023]

[7] D. Wu., et al., Characteristics of $\mathrm{X} /$-ray radiations by intense laser interactions with high- $\mathrm{Z}$ solids: The role of bremsstrahlung and radiation reactions, Matter and Radiation at Extremes 3.6 (2018) 293-299

[https://doi.org/10.1016/j.mre.2018.06.002]

[8] C. D. Armstrong, et al., Bremsstrahlung emission profile from intense laser-solid interactions as a function of laser focal spot size, Plasma Physics and Controlled Fusion 61.3 (2019) 034001

[https://doi.org/ 10.1088/1361-6587/aaf596]

[9] G. Hernández and F. Fernández. Diagnostic of electron temperature from bremsstrahlung in overdense targets, Applied Physics B 124.7 (2018) 135

[https://doi.org/10.1007/s00340-018-6999-5]

[10] R. A. Snavely et al., Intense high-energy proton beams from petawatt-laser irradiation of solids, Phys. Rev. Lett. 85 (2000) 2945-2948

[https://doi.org/10.1103/PhysRevLett.85.2945]

[11] A. Maksimchuk, et al., Forward ion acceleration in thin films driven by a high-intensity laser, Phys. Rev. Lett. 84 (2000) 4108-4111

[https://doi.org/10.1103/PhysRevLett.84.4108]

[12] D. R. Rusby et al., Pulsed x-ray imaging of high density objects using a ten picosecond high-intensity laser driver, Emerging Imaging and Sensing Technologies, Vol. 9992. International Society for Optics and Photonics, 2016

[https://doi.org/10.1117/12.2241776]

[13] Y. Glinec, et al. High-resolution -ray radiography produced by a laser-plasma driven electron source, Phys. Rev. Lett. 94(2) (2005) 025003 
[https://doi.org/10.1103/PhysRevLett.94.025003]

[14] Y. A. Gauduel, Laser-plasma accelerator and femtosecond photon sources-based ultrafast radiation chemistry and biophysics, JINST 12(02) (2017) C02048

[https://doi.org/10.1088/1742-6596/373/1/012012]

[15] K. Nakajima, et al. Laser-driven very high energy electron/photon beam radiation therapy in conjunction with a robotic system. Applied Sciences, 5(1) (2015) 1-20

[https://doi.org/10.3390/app5010001]

[16] A. L. Meadowcroft and R. D. Edwards, High-Energy Bremsstrahlung Diagnostics to Characterize HotElectron Production in Short-Pulse Laser-Plasma Experiments, IEEE Transactions on Plasma Science, 40.8 (2012) 1992-2001

[https://doi.org/10.1109/TPS.2012.2201175]

[17] S. Palaniyappan, et al. MeV bremsstrahlung X rays from intense laser interaction with solid foils. Laser and Particle Beams, 36(LA-UR-18-25214), 2018

[https://doi.org/10.1017/S0263034618000551]

[18] B. Borm, et al., Properties of laser-driven hard x-ray sources over a wide range of laser intensities. Physics of Plasmas, 26(2) (2019) 023109

[https://doi.org/10.1063/1.5081800]

[19] G. J. Williams, et al, High-energy differential-filtering photon spectrometer for ultraintense laser-matter interactions. Review of Scientific Instruments, 89(10) (2018) 10F116

[https://doi.org/10.1063/1.5039383]

[20] D. Haden, et al., High energy X-ray Compton spectroscopy via iterative reconstruction, Nuclear Instruments and Methods in Physics Research Section A: Accelerators, Spectrometers, Detectors and Associated Equipment, 951 (2020) 163032

[10.1016/j.nima.2019.163032]

[21] S. Singh, et al., Compact high energy x-ray spectrometer based on forward Compton scattering for high intensity laser plasma experiments, Review of Scientific Instruments 89.8 (2018) 085118

[https://doi.org/10.1063/1.5040979]

[22] R. Scott, et al. Measuring fast electron spectra and laser absorption in relativistic laser-solid interactions using differential bremsstrahlung photon detectors. Review of Scientific Instruments, 84(8) (2013) 083505

$$
\text { [https://doi.org/10.1063/1.4816332] }
$$

[23] FUJI film official website: [http://www.fujifilm.com/]

[24] S. Singh, et al., Absolute calibration of imaging plate detectors for electron kinetic energies between $150 \mathrm{keV}$ and $1.75 \mathrm{MeV}$, Rev. Sci. Instrum. 88 (2017) 075105

[https://doi.org/10.1063/1.4993921] 
[25] F. Consoli et al., Laser produced electromagnetic pulses: generation, detection and mitigation. High Power Laser Science and Engineering 8 (2020)

[https://doi.org/10.1017/hpl.2020.13]

[26] S. G. Gales \& C. D. Bentley, Image plates as x-ray detectors in plasma physics experiments. Review of scientific instruments, 75(10) (2004) 4001- 4003

[https://doi.org/10.1063/1.1789256]

[27] ELI Beamlines official website: [https://www.eli-beams.eu/]

[28] D. R. Rusby, et al., Novel scintillator-based x-ray spectrometer for use on high repetition laser plasma interaction experiments. Review of Scientific Instruments, 89(7) (2018) 073502.

[https://doi.org/10.1063/1.5019213]

[29] J. Vyskocil, O. Klimo, and S. Weber. Simulations of bremsstrahlung emission in ultra-intense laser interactions with foil targets, Plasma Physics and Controlled Fusion, 60(5) (2018) 05401

[https://doi.org/10.1088/1361-6587]

[30] F. Wan, et. al. Photon emission by bremsstrahlung and nonlinear Compton scattering in the interaction of ultraintense laser with plasmas, The European Physical Journal D, 71(9) (2017) 236

[https://doi.org/10.1140/epjd/e2017-70805-7]

[31] K. T. Phuoc, et al., All-optical Compton gamma-ray source, Nat Photonics 6 (2012) 308-311

[https://doi.org/10.1038/nphoton.2012.82]

[32] O. N. Rosmej, et al., Generation of keV hot near-solid density plasma states at high contrast laser-matter interaction, Physics of Plasmas, 25(8) (2018) 083103

[https://doi.org/10.1063/1.5027463]

[33] C. Zulick, et al., High resolution bremsstrahlung and fast electron characterization in ultrafast intense laser-solid interactions. New Journal of Physics, 15(12) (2013) 123038

[https://doi.org/10.1088/1367-2630]

[34] M. R. Kaçal, F. Akman, and M. I. Sayyed. Evaluation of gamma-ray and neutron attenuation properties of some polymers, Nuclear Engineering and Technology, 51.3 (2019) 818-824

[https://doi.org/10.1016/j.net.2018.11.011]

[35] FLUKA official website: [https://fluka.cern]

[36] G. Battistoni, et al., Overview of the FLUKA code, Annals of Nuclear Energy 82 (2015) 10-18 [https://doi.org/10.1016/j.anucene.2014.11.007]

[37] T.T. Bohlen, et al., The FLUKA Code: Developments and Challenges for High Energy and Medical Applications, Nuclear Data Sheets 120 (2014) 211-214

[https://doi.org/10.1016/j.nds.2014.07.049] 
[38] V. Vlachoudis. FLAIR: A Powerful But User Friendly Graphical Interface For FLUKA, in Proc. Int. Conf. on Mathematics, Computational Methods \& Reactor Physics (M\&C 2009), Saratoga Springs, New York, 2009

[39] Y. Q. Cui, et al., Laser absorption and hot electron temperature scalings in laser - plasma interactions. Plasma Physics and Controlled Fusion, 55(8) (2013) 085008

[https://doi.org/10.1088/0741-3335/55/8/085008]

[40] A. G. Mordovanakis, et al., Temperature scaling of hot electrons produced by a tightly focused relativistic-intensity laser at $0.5 \mathrm{kHz}$ repetition rate, Applied Physics Letters, 96(7) (2010) 071109

[https://doi.org/10.1063/1.3306730]

[41] Y. Dodge. The Concise Encyclopedia of Statistics. Springer, 2008

[42] D. Margarone, et al., A laser-driven ion accelerator for multidisciplinary applications, Quantum Beam Science, 2(2) (2018) 8

[https://doi.org/10.3390/qubs2020008]

[43] P. Krist \& J. Bila, A mathematical model of the MT 25 microtron, JINST 6(10) (2011) T10005

[https://doi.org/10.1063/1.4739404]

[44] M. Tryus et al., TERESA Target Area at ELI Beamlines, Quantum Beam Science, 4(4) (2020) 37

[https://doi.org/10.3390/qubs4040037]

[45] E. Sistrunk, et al., All diode-pumped, high-repetition-rate advanced petawatt laser system (HAPLS), CLEO: Science and Innovations. Optical Society of America, 2017 C. STh1L. 2

[https://doi.org/10.1364/CLEO_SI.2017.STh1L.2]

[46] T. Kluge et al., Electron temperature scaling in laser interaction with solids, Physical Review Letters 107(20) (2011) 205003

[https://doi.org/10.1103/PhysRevLett.107.205003]

[47] S. C. Wilks, et al., Energetic proton generation in ultra-intense laser-solid interactions., Physics of Plasmas, 8 (2001) 542

[https://doi.org/10.1063/1.1333697]

[48] K. Jungwirth, et al., The Prague asterix laser system, Physics of Plasmas 8 (2001) 2495-2501

[https://doi.org/10.1063/1.1350569]

[49] S. Singh, et al., Bremsstrahlung emission and plasma characterization driven by moderately relativistic laser-plasma interactions, Plasma Physics and Controlled Fusion, 2020 (under review).

[50] M. Krupka, et al. Comprehensive diagnostics of hot electron emission from plasmas produced by subnanosecond terawatt laser on thin foil metal targets. JINST 14(12) (2019) C12003

[https://doi.org/10.1088/1748-0221/14/12/C12003] 
[51] M. Krupka, S. Singh, T. Pisarczyk et al., "Design of modular multi-channel electron spectrometers for application in laser matter interaction experiments at Prague Asterix Laser System", Review of Scientific Instruments, 2020 (under review).

[52] M. Huault, et al., A 2D scintillator-based proton detector for high repetition rate experiments, High Power Laser Science and Engineering 7 (2019)

[https://doi.org/10.1017/hpl.2019.43]

[53] G. A. P. Cirrone, et al., Clinical and research activities at the CATANA facility of INFN-LNS: From the conventional hadrontherapy to the laser-driven approach, Frontiers in Oncology 7 (2017) 223

[https://doi.org/10.3389/fonc.2017.00223] 